\title{
Solid contact ion selective electrodes (ISE) for applications in life sciences, biotechnology and environmental monitoring - Technology and Performance
}

\author{
Christoph Bieg, Kai Fuchsberger, Gorden Link, Ramona Samba, Simon Werner, Martin Stelzle ${ }^{1}$ \\ NMI Natural and Medical Sciences Institute at the University of Tübingen, Germany \\ martin.stelzle@nmi.de
}

\begin{abstract}
Multiple applications exist for low-cost solid-contact ion selective electrodes (sc-ISE) in medicine, biotechnology, environmental monitoring, and food production. The objective of this study is to provide technology and demonstrate proof of concept for solid contact sensors that are sensitive, selective, allow for sterilization, exhibit long shelf life and short run-in time, and are biocompatible, biostable and possibly even allow for calibration free operation or in situ recalibration, respectively. We present a novel solid contact technology based on hydrophobic carbon formulations and demonstrate enhanced stability and reduced spread and drift of electrode potentials obtained thereby. Integration of sensor arrays with microfluidics yields a sensor probe intended for multi-modal detection requiring only very small sample volume. In addition, in situ recalibration enabled by microfluidics is anticipated to enable long-term unattended operation in the future.
\end{abstract}

(Suppl. Info: https://datenwolke.nmi.de/index.php/s/cn4XKPozC49p7cJ)

Key words: solid contact, ion selective electrodes, ion-to-electron transducer,

\section{Introduction}

Multiple applications would exist for low-cost solid-contact ISE (sc-ISE) in medicine, biotechnology, and environmental/agriculture [1]. Conventional glass ion selective electrodes in contrast do not allow for dry storage, are relatively expensive and fragile, and require frequent recalibration. To this end, low cost, dry storage, fast run-in, long-term stable, biocompatible, low drift, multi-modal solid-state sensors would be highly desirable. Currently available solid-contact ISE typically exhibit relatively large drift and lack reliable reference electrodes. As a fundamental cause, the formation of a water layer due to hydrophilic properties of the ion-to-electron transducer (i2eT) has been identified [2]. Towards finding a remedy for this problem, mesoporous carbon materials have drawn considerable attention as they are hydrophobic and exhibit a high specific surface and capacitance even at small electrode size [3], [4]. Employing this technology in combination with a microfluidic sampling and recalibration scheme, a sensorprobe was devised comprising a sensor array with the novel solid-contact possibly enabling single point re-calibration so that long term and unattended operation would become conceivable.

\section{Results \\ Ion-to-electron transducer}

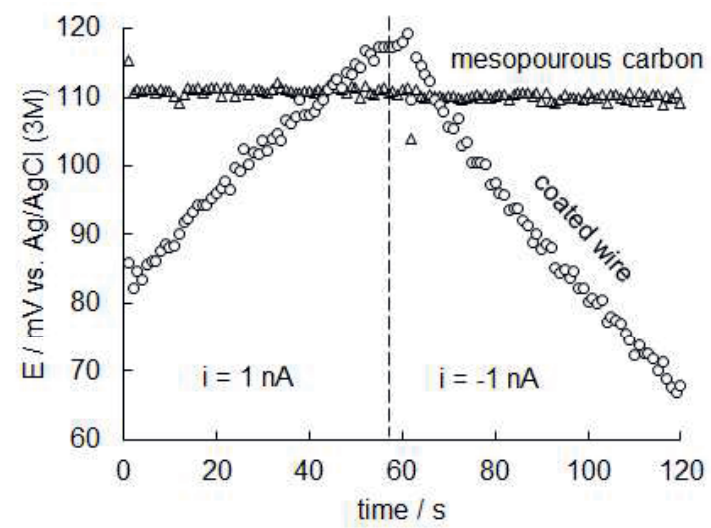

Fig. 1. Comparison of Chronopotentiograms of $\mathrm{pH}$ ISE based on coated wire technology (circles) vs. solid contacts based on hydrophobic mesoporous carbon material (triangles). Virtually no change of potential is observed with the latter electrode upon challenge with a current of $+/-1 n A$ indicating enhanced potential stability. 
A novel solid contact technology is employed, based on mesoporous carbon compounds exhibiting extremely low concentration of hydrophilic surface moieties. Electrodes using this solid-contact material exhibit significantly improved potential stability as is demonstrated by the Chronopotentiograms [5] (Fig.1, Tab.1). This observation may be explained by the large capacitance of this material.

Tab. 1: Capacitance determined by the current test method with the coated wire configuration (membrane on metal) versus mesoporous carbon as ion-to-electron transducer.

\begin{tabular}{|c|c|}
\hline $\mathrm{pH}-\mathrm{ISE}$ & $\mathrm{C}_{\mathrm{L}} / \mu \mathrm{F}$ \\
\hline coated wire & 1,26 \\
\hline mesoporous carbon & 339,8 \\
\hline
\end{tabular}

\section{Comparison of Iridiumoxide (IrOx) and C}

Potential stability of Sc-ISE depends critically on the performance of the ion-to-electron transducer, i.e. its ability to store charge due to current inevitably flowing during measurement of the electrode potential without notable variation of this potential. With IrOx this is achieved via redox reactions occurring within this material whereas the carbon material stores charge due to an extremely large specific interfacial capacitance.

Tab. 2: Comparison of spread of initial potential after first immersion into buffer solution of two ion-toelectron-transducer materials: iridium oxide and mesopourous carbon in $\mathrm{pH}$-SC-ISE.

\begin{tabular}{|c|c|c|}
\hline $\mathrm{pH}-\mathrm{ISE}$ & $\begin{array}{c}\text { Offset SD } \\
\text { at } \mathrm{pH} 7 / \\
\mathrm{mV}\end{array}$ & $\begin{array}{c}\text { Drift in PBS } \\
/ \mathrm{mV} \cdot \mathrm{h}^{-1} \pm \\
\mathrm{SD}(\mathrm{n}=3)\end{array}$ \\
\hline $\begin{array}{c}\text { IrOx on gold } \\
\text { electrodes }\end{array}$ & 55 & $1,44 \pm 0,83$ \\
\hline $\begin{array}{c}\text { Mesoporous } \\
\text { Carbon on Glassy } \\
\text { Carbon } \\
\text { electrodes }\end{array}$ & 3,48 & $0,02 \pm 0,01$ \\
\hline
\end{tabular}

In addition, spread of the initial potential measured after immersing electrodes into a buffer solution was shown to be much smaller in case of the electrodes using mesoporous carbon as solid contact when compared to those based on the IrOx contact (Tab.2). This is a very attractive feature as it would enable single-use electrodes ready-to-use without prior calibration. Additionally, potential drift is reduced as well in hydrophobic carbon based i2eT when compared to IrOx.

The higher potential drift found with IrOx based i2eT can be ascribed to the formation of a water layer at the interface between the ISEmembrane and the hydrophilic IrOx i2eT. This formation of a water layer is strongly suppressed in hydrophobic carbon based i2eT.

\section{Calibration of SC-ISE}

Sc-ISE were manufactured based on our novel solid contact technology for three different analytes typically called for in applications in medicine and biotechnology, namely pH (Fig.2), $\mathrm{K}^{+}$(Fig.3), and $\mathrm{NH}_{4}{ }^{+}$(Fig.4).

All electrodes show excellent Nernst type behavior, very good sensitivity, and low spread of initial electrode potential.

The $\mathrm{pH}$ electrodes are useful for measurement between $\mathrm{pH} 3$ and 10, much larger than optical solid state measurement technologies.

These results are summarized in Tab. 3 .

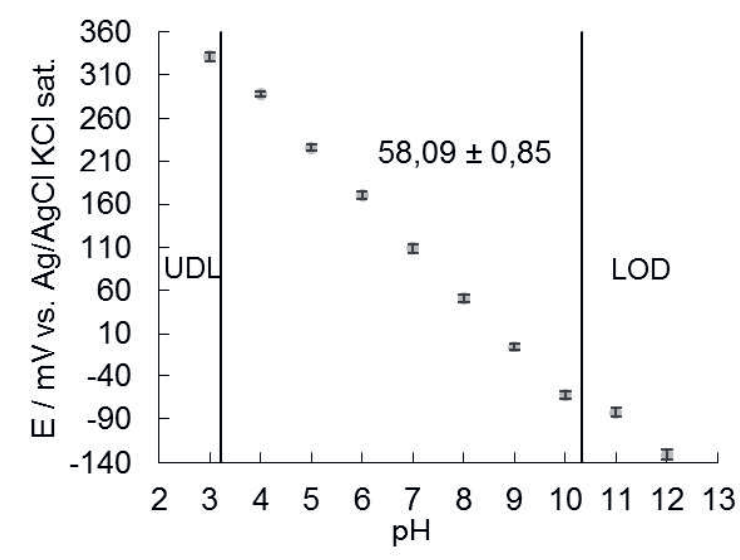

Fig. 2. Calibration of $\mathrm{pH}$-sensors ( $n=9)$ comprised of modified carbon transducer and PVC based ISEmembrane. SD Offset $< \pm 5 \mathrm{mV}$. 


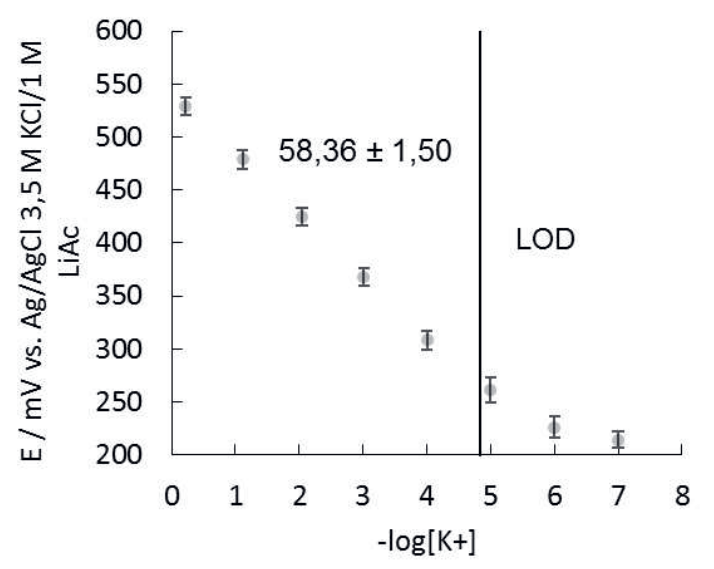

Fig. 3. Calibration of $\mathrm{K}^{+}$-sensors $(n=8)$ comprised of modified carbon transducer and PVC based ISEmembrane. $S D$ Offset at $1 \mathrm{mM} \pm 8.12 \mathrm{mV}$.

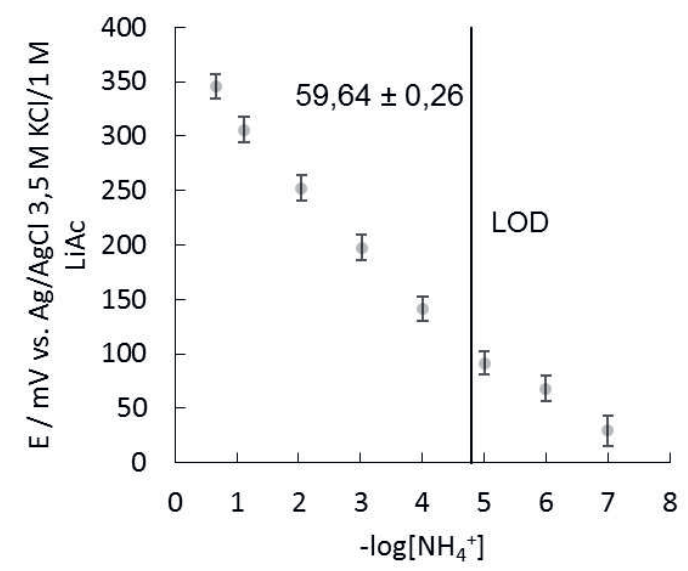

Fig. 4. Calibration of $\mathrm{NH}_{4}^{+}$-sensors ( $\left.n=6\right)$ comprised of modified carbon transducer and PVC based ISE-membrane. SD Offset at $1 \mathrm{mM} \mathrm{NH} 4 \mathrm{Cl} \pm$ $11.40 \mathrm{mV}$.

Tab. 3: Calibration Data for $\mathrm{pH}, \mathrm{K}^{+}$and $\mathrm{NH}_{4}^{+}-$ISE.

\begin{tabular}{|l|l|l|l|l|l|l|}
\hline ISE & $\begin{array}{l}\text { slope / } \\
\mathrm{mV} \cdot \mathrm{dec}^{-1} \pm \\
\mathrm{SD}\end{array}$ & $\begin{array}{l}\text { Offset } \\
\mathrm{SD} \text { at } \\
\mathrm{pH} \mathrm{7} \mathrm{or} \\
1 \mathrm{mM} \\
\mathrm{MCl} / \pm \\
\mathrm{mV}\end{array}$ & $\begin{array}{l}\text { Drift in 1 } \\
\mathrm{mM} \mathrm{KCl} / \\
\mathrm{mV} \cdot \mathrm{h}^{-1} \pm \\
\mathrm{SD}\end{array}$ & $\begin{array}{l}\text { Hysteresis / } \\
\mathrm{mV} \pm \mathrm{SD}\end{array}$ & $\begin{array}{l}\text { LOD / - } \\
\log \left(\mathrm{a}\left(\mathrm{K}^{+}\right) \pm \mathrm{SD}\right.\end{array}$ & $\begin{array}{l}\text { UDL / - log }\left(\mathrm{a}\left(\mathrm{K}^{+}\right)\right. \\
\pm \mathrm{SD}\end{array}$ \\
\hline $\mathrm{pH}$ & $58,09 \pm 0,85$ & 3,48 & $0,02 \pm 0,01$ & $2,77 \pm 2,10$ & $10,31 \pm 0,05$ & $3,22 \pm 0,11$ \\
\hline $\mathrm{K}^{+}$ & $58,36 \pm 1,50$ & 8,12 & n.d. & $4,54 \pm 6,65$ & $4,84 \pm 0,08$ & $>0$ \\
\hline $\mathrm{NH}_{4}^{+}$ & $59,64 \pm 0,26$ & 11,40 & $0,14 \pm 0,07$ & $1,88 \pm 0,87$ & $4,80 \pm 0,03$ & $>0$ \\
\hline
\end{tabular}

\section{Sensor probe with in-situ recalibration}

A sensor-probe (Fig.5) was developed comprising four independent solid-contact ISE, an amperometric sensor, an impedance sensor and three $\mathrm{Ag} / \mathrm{AgCl}$ reference electrodes integrated with an arrangement of microfluidic channels, which enable sample acquisition and analysis as well as in situ recalibration by rinsing a calibration buffer over the electrodes by means of external pumps. Sample volume required for a measurement is about 100 $200 \mu \mathrm{l}$.

This system should be applicable in applications requiring long term monitoring of an array of parameters such as $\mathrm{pH}$, oxygen content, impedance and electrolytes. Such applications exist in medicine, biotechnology, environmental monitoring, and agriculture.

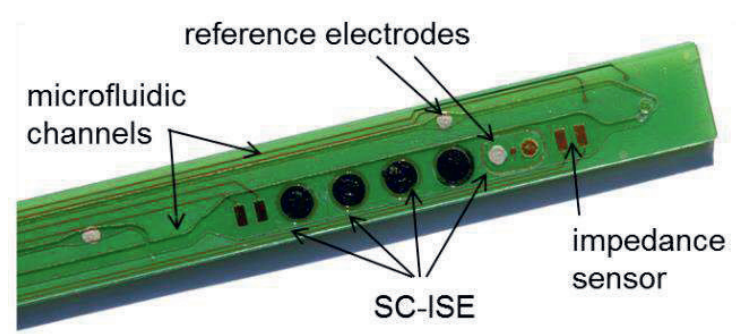

Fig. 5: Sensor probe comprising sc-ISE, amperometric sensor, impedance sensor and reference electrodes integrated with microfluidic 
channels to enable sample acquisition and in situ recalibration.

\section{Evaluation of SC-ISE in real-world sample matrices}

A pH electrode was tested in the context of a biofermentation experiment. The electrode was calibrated by a single point calibration at $\mathrm{pH} 7$ and subsequently immersed in fermentation broth along with a commercial $\mathrm{pH}$ electrode (calibrated by a standard two-point calibration prior to the measurement). As displayed in Fig.6, both electrodes determine very similar $\mathrm{pH}$ values throughout the entire duration of the fermentation experiment. This means that the sensitivity (slope) of the sc-pH electrode is maintained over 30 days (Fig.6) allowing for simple single point calibration of this electrode, which for example is easily achieved by addition of fresh medium to a fermentation broth.

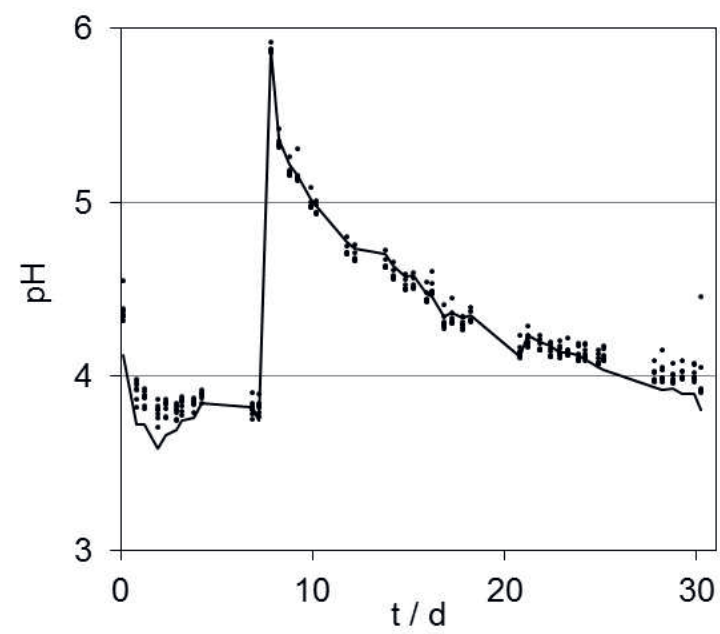

Fig. 6: Comparative measurement of $\mathrm{pH}$-sc-ISE (dots) $(\mathrm{N}=7)$ and commercial glass $\mathrm{pH}$ electrode (line) in yeast fermentation process over 30 days.

\section{Conclusion}

Enhanced performance of novel solid-contact ion-to-electron transducer based on mesoporous carbon materials was demonstrated. Integration of a sensor array with microfluidic probe technology enables multimodal sensing, use of conventional solid state $\mathrm{Ag} / \mathrm{AgCl}$ reference electrodes, re-calibration and potential use in long-term monitoring applications.

Future research will aim for the integration of a chloride insensitive sc-reference electrode with sc-ISEs and applications development for medicine, biotechnology, and environmental lagricultural sensing.

\section{Acknowledgements}

Funding for this study was partially obtained from the BMBF through grants no. 16SV6026 and 13XP5009B. Helpful discussions with W. Künnecke and M. Hartlep are acknowledged.

\section{References}

[1] C. Bieg, K. Fuchsberger, M. Stelzle, Introduction to polymer-based solid-contact ion-selective electrodes-basic concepts, practical considerations, and current research topics, Analytical and Bioanalytical Chemistry, 1-17.(2016), doi: doi:10.1007/s00216-016-9945-6.

[2] M. Fibbioli, W. E. Morf, M. Badertscher, N. F. de Rooij, E. Pretsch, Potential Drifts of Solid-Contacted Ion-Selective Electrodes Due to Zero-Current lon Fluxes Through the Sensor Membrane, Electroanalysis, 12, 1286-1292.(2000), doi:

[3] B. Paczosa-Bator, lon-selective electrodes with superhydrophobic polymer/carbon nanocomposites as solid contact, Carbon, 95, 879-887.(2015), doi: http://dx.doi.org/10.1016/j.carbon.2015.09. 006.

[4] J. Hu, X. U. Zou, A. Stein, P. Bühlmann, Ion-selective electrodes with colloidimprinted mesoporous carbon as solid contact, Analytical Chemistry, 86, 71117118.(2014), doi: 10.1021/ac501633r.

[5] J. Bobacka, Potential stability of all-solidstate ion-selective electrodes using conducting polymers as ion-to-electron transducers, Analytical Chemistry, 71, 4932-4937.(1999), doi: 10.1021/ac990497z. 